\title{
The prevalence of glaucoma in patients undergoing surgery for eyelid entropion or ectropion
}

\author{
This article was published in the following Dove Press journal: \\ Clinical Interventions in Aging \\ 12 October 2016 \\ Number of times this article has been viewed
}

\section{Shani Golan \\ Gilad Rabina \\ Shimon Kurtz \\ Igal Leibovitch}

Division of Orbital and Ophthalmic Plastic Surgery, Department of Ophthalmology, Tel Aviv Medical Center, Sackler Faculty of Medicine, Tel Aviv University, Tel Aviv, Israel
Correspondence: Shani Golan Division of Orbital and Ophthalmic Plastic Surgery, Department of Ophthalmology, Tel Aviv Medical Center, Sackler Faculty of Medicine, 6 Weizman Street, Tel Aviv 64239, Israel

Tel +97236973408

Fax +972 36973867

Email shanigola2@gmail.com
Purpose and design: The aim of this study was to establish the prevalence of known glaucoma in patients undergoing ectropion or entropion surgical repair. In this study, retrospective review of case series was performed.

Participants: All patients who underwent ectropion or entropion surgery in a tertiary medical center between 2007 and 2014 were included. The etiology of eyelid malpositioning was involutional or cicatricial.

Methods: The medical files of the study participants were reviewed for the presence and type of glaucoma, medical treatment, duration of treatment, and the amount of drops per day. These data were compared to a matched control group of 101 patients who underwent blepharoplasty for dermatochalasis in the same department during the same period.

Main outcome measure: In this study, the prevalence of glaucoma in individuals with ectropion or entropion was the main outcome measure.

Results: A total of 227 patients (57\% men, mean age: 79.2 years) who underwent ectropion or entropion surgery comprised the study group and 101 patients who underwent upper blepharoplasty for dermatochalasis comprised the control group. Compared to four patients in the control group (4\%, $P=0.01), 30$ of the study patients $(13.2 \%)$ had coexisting glaucoma. Of 30 glaucomatous patients, 25 had primary open-angle glaucoma for a mean duration of 10.3 years. The glaucomatous patients were treated with an average of 2.7 antiglaucoma medications.

Conclusion: An increased prevalence of known glaucoma in patients undergoing ectropion or entropion repair surgery was found. This observation may indicate that the chronic usage of topical anti-glaucoma eyedrops may lead to an increased risk of developing eyelid malpositions, especially in elderly patients.

Keywords: cicatricial, lamella, review

\section{Introduction}

Ectropion and entropion in elderly patients are usually assumed to be involutional. Involutional changes include lower eyelid laxity, disinsertion of the retractor muscles (capsulopalpebral and inferior tarsal muscles), canthal ligament weakness, and overriding orbicularis muscle in entropion. ${ }^{1}$ Cicatricial ectropion occurs from scarring of the anterior lamella by conditions such as facial burns, trauma, chronic dermatitis, or excessive skin excision during surgery. ${ }^{1}$

The treatment of most forms of glaucoma includes the use of topical agents that enhance aqueous humor outflow, reduce aqueous production, or both. ${ }^{2}$ The list of side effects of these agents is long and includes eyelid dermatitis, lacrimal system scarring, ocular discomfort upon instillation, tear film instability, conjunctival inflammation, subconjunctival fibrosis, conjunctival epithelium changes, and corneal surface and endothelial impairment. ${ }^{2}$ 
A review of the literature over the past 15 years yielded only three case reports ${ }^{3-5}$ and one case series ${ }^{6}$ of ectropion or entropion purportedly induced by antiglaucoma medications. These reports found a correlation between the usage of antiglaucoma medications and the development of eyelid malposition abnormalities, but there were very few patients to establish the prevalence of the coexistence of these ophthalmologic pathologies. This retrospective analysis was conducted to establish the prevalence of glaucoma in patients with either involutional or cicatricial ectropion or entropion undergoing eyelid repair.

\section{Methods}

This is a retrospective chart review of patients who underwent surgery in the medical center for ectropion or entropion between 2007 and 2014. The institutional review board at Tel Aviv Medical Center approved this study. The review board advised patient consent was not required as this is a retrospective case series study. All patients underwent a complete oculoplastic and ophthalmological examination, including the assessment of horizontal laxity (using the snapback test), the degree of retractor disinsertion (inferior fornix depth, lower eyelid movement, or down gaze), and the degree of superior migration of the preseptal orbicularis (as the patients squeeze their eyes closed).

Patients with a symptomatic entropion (eyelid margin eversion, causing ocular irritation or corneal rubbing) or symptomatic ectropion (outward turning of the lower eyelid, causing dry eye symptoms) were referred to surgery. Ectropion and entropion were subclassified as involutional (age-related weakness of canthal ligaments, disinsertion of retractors) or cicatricial (scarring or shortening of the anterior or posterior lamella). Patients with paralytic, mechanical, or congenital eyelid malposition were excluded.

The demographic data and pertinent medical history, as well as the presence of known glaucoma and other ocular and systemic diseases were retrieved and recorded. Primary open-angle glaucoma (POAG) was defined according to the guidelines of European Glaucoma Society. ${ }^{7}$ An eye was considered as being affected by POAG when the following criteria were fulfilled: 1) open angle at gonioscopy, 2) intraocular pressure $>21 \mathrm{mmHg}$ with no treatment, 3) typical abnormal optic nerve head and/or typical glaucomatous visual field loss, and 4) no evidence of apparent secondary cause of glaucoma. Pseudoexfoliation glaucoma was diagnosed when the criteria for POAG coexisted with pseudoexfoliation as being documented on ophthalmic examination.

Chronic angle-closure glaucoma was defined as: 1) iridocorneal apposition/adhesions, 2) chronic rise in intraocular pressure, 3) despite an iridotomy, and 4) glaucomatous optic neuropathy.

The type of antiglaucoma medications was subdivided into different subgroups of treatments (ie, beta antagonists, carbonic anhydrase inhibitors, and prostaglandin analogs), and the treatment duration and the amount of drops being instilled per day were also recorded. The collective data on the study patients were compared to the same findings in patients who underwent blepharoplasty for dermatochalasis, which was defined as excess skin obscuring the upper visual field, during the study period (control group).

\section{Statistical analysis}

Data were recorded in Microsoft Excel and analyzed using SPSS Version 21 (IBM Corporation, Armonk, NY, USA). Comparison between numerical variables, such as age, and between patients undergoing ectropion or entropion repair and patients in the control group was performed using independent sample $t$-test. For nominal parameters such as sex and the presence of glaucoma, Pearson's chi-square test was used, while for ordinal or not normally distributed variables, Mann-Whitney nonparametric test was used.

\section{Results}

Of the 252 patients who underwent ectropion or entropion surgery in the Tel Aviv Medical Center, 23 patients had died or their records were missing, and two patients were known to have glaucoma but their records were inadequate: these 25 patients were excluded from the study, and the remaining 227 patients who had undergone ectropion or entropion surgery were enrolled in the study. A total of 115 patients had undergone ectropion repair surgery (Figures 1 and 2). Of them 87 (76\%) had involutional ectropion and 28 (24\%) had cicatricial ectropion. The remaining 112 patients had an involutional entropion repair surgery (Figure 3).

The control group comprised 101 patients with dermatochalasis who underwent upper blepharoplasty surgery (Table 1).

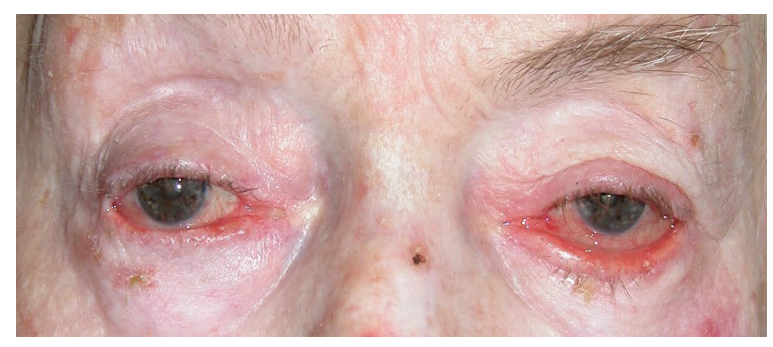

Figure I Severe left lower lid ectropion in an 80-year-old patient treated with Cosopt (dorzolamide hydrochloride-timolol maleate) and Alphagan (alpha 2 agonists) for 10 years. 


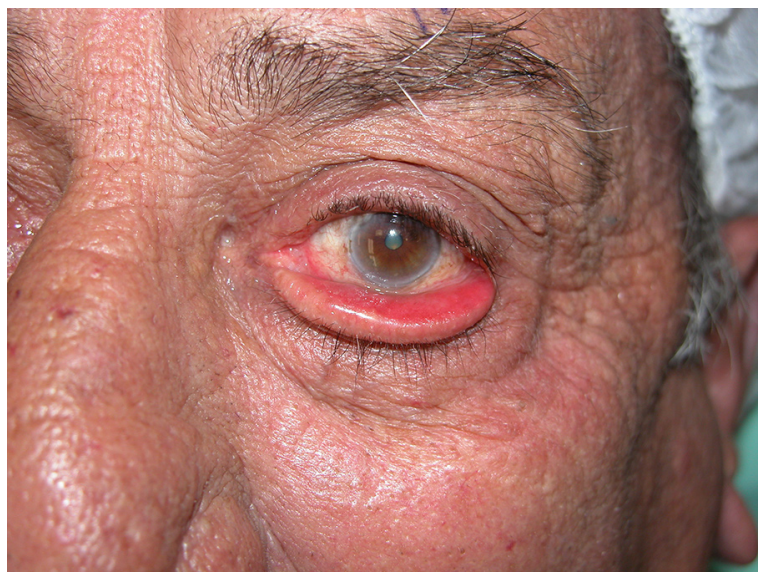

Figure 2 Severe left lower lid ectropion in a 78-year-old patient treated with Xalatan (prostaglandin analog) for 5 years.

There were significantly fewer women in the study group (43\%) than in the control group $(64 \%, P=0.002)$. The mean age of the study patients had a trend but was not significantly higher than that of the controls (79.2 years compared to 77.6 years, respectively, $P=0.053)$. Compared to four patients in the control group ( $4 \%, P=0.01), 30$ patients $(13.2 \%)$ in the study group were diagnosed as having glaucoma (14 patients with ectropion and 16 with entropion, $P=0.47$ ). Out of the 14 patients who had undergone ectropion surgery and were diagnosed with glaucoma, 13 had involutional ectropion and one had cicatricial ectropion.

Of the 30 glaucomatous patients, 25 had POAG with a mean duration of 10.3 years (range: 1-21 years), and the four glaucomatous controls had POAG with a mean duration of 9.25 years. None of them had chronic angle-closure glaucoma. The glaucomatous study patients were treated with an average of 2.7 antiglaucoma medications, most commonly a combination of dorzolamide hydrochloride (carbonic anhydrase inhibitor)-timolol maleate (beta antagonist) and latanoprost (prostaglandin analog). The four glaucomatous patients in the control group were treated with a combination of dorzolamide hydrochloride-timolol maleate.

\section{Discussion}

The paucity of reported patients has precluded the establishment of the prevalence of known glaucoma among patients

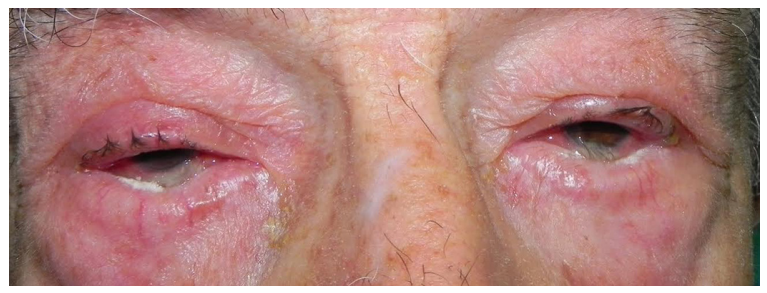

Figure 3 Bilateral eyelid dermatitis and lower lid entropion in a 90 -year-old male patient treated with Cosopt and Lumigan (prostaglandin analog) in both eyes for 4 years.
Table I Patient's basic demographic data in the study (ectropion/ entropion) and control (dermatochalasis) groups

\begin{tabular}{lll}
\hline Characteristics & Study group & Control group \\
\hline Female/male & $98 / 129$ & $64 / 37$ \\
Mean age (years) & 79.2 & 77.6 \\
$\quad$ Ectropion/entropion & $115 / 112$ & $\mathrm{NA}$ \\
$\quad \begin{array}{l}\text { Involutional/cicatricial ectropion } \\
\text { Number of patients diagnosed }\end{array}$ & $87 / 28$ & $\mathrm{NA}$ \\
$\begin{array}{c}\text { As having glaucoma (type) } \\
\text { Ectropion/entropion }\end{array}$ & $30(25 \mathrm{POAG}$, & 4 (POAG) \\
\hline
\end{tabular}

Abbreviations: POAG, primary open-angle glaucoma; PXFG, pseudoexfoliation glaucoma; NA, not applicable.

with ectropion or entropion. The authors reviewed a database of a relatively large number of patients with eyelid malpositions and found a $13.2 \%$ prevalence of treated glaucoma (treated with antihypertensive eyedrops) among them. This prevalence is higher than the overall prevalence of POAG of $6.8 \%$ in the general population (increasing from $2.16 \%$ in white women aged $70-74$ years to $6.94 \%$ in those 80 years and older). ${ }^{8}$ This prevalence also exceeds the $4 \%$ that was found in the control patients who were undergoing blepharoplasty $(P=0.01)$.

Ectropion and entropion in elderly patients is most commonly involutional, and the etiologies are increased horizontal and vertical lid laxity. ${ }^{1,9-12}$ Horizontal lid laxity is related to involutional changes in the tarsal plate and orbicularis muscle (canthal tendons stretching expressed by the medial canthal tendon laxity). ${ }^{1,9-12}$ Vertical lid laxity is related to involutional changes described as attenuation, dehiscence, or disinsertion of the lower lid retractors and orbital septum, and it is characterized by an alteration of lower lid excursion. ${ }^{1,12}$

The treatment of most forms of glaucoma includes the use of topical agents that enhance aqueous humor outflow, reduce aqueous production, or both. ${ }^{2}$ Topical anti-ocular hypertensive drugs must penetrate across the tissues of the eye to reach their therapeutic targets. These tissues are often the first to show signs and symptoms of drug toxicity and adverse effects. There are few reports that address the prevalence of eyelid malpositions in patients treated with intraocular pressure-lowering eyedrops. ${ }^{3-6,13}$ Hegde et al reported a retrospective analysis of 13 patients who were treated with topical medications and presented with topical drug-induced ectropion. ${ }^{6}$ Dorzolamide (carbonic anhydrase inhibitor) was the most common offending agent (53\%), followed by brimonidine (alpha 2 agonists; 23\%). Those authors suggested that the chronic exposure to the causative agent leads to cicatricial changes in the anterior lamella of the eyelid in susceptible individuals and that this can manifest 
as contact dermatitis leading to tissue edema and mechanical ectropion. On the other hand, Altieri and Ferrari ${ }^{13}$ did not find any sequelae, such as increased laxity or disruption of eyelid tissues, after the use of the topical ophthalmic prostaglandin analogs such as latanoprost, travoprost, and bimatoprost. This study reveals that $73 \%$ (22/30) of the patients were treated with a combination of dorzolamide hydrochloride-timolol and $63 \%(19 / 30)$ were treated with prostaglandin analog.

The observation can potentially be explained by two main mechanisms: 1) involutional pathogenesis, in which the chronic usage of ocular eyedrops causes mechanical stretching of the eyelid, thereby increasing horizontal and vertical lid laxity and eventually causing eyelid malposition and drug hypersensitivity. ${ }^{1}$ This explanation is further supported by the observation that $13 / 14$ patients with ectropion who were diagnosed with glaucoma had involutional ectropion; 2) any allergic or toxic skin reaction may cause tissue edema, which, in turn, enhances eyelid laxity, with the skin cicatrization eventually causing anterior lamellar shortening and ectropion or entropion.

There was a higher percentage of males in the study group compared to the control group $(P=0.002)$. This difference is consistent with earlier studies that demonstrated higher percentage of men undergoing ectropion surgeries. ${ }^{14}$

There are some limitations to this study associated with its retrospective design and the fact that only patients with symptomatic ectropion or entropion who underwent surgery were included and not the patients with either of these disorders who did not have surgery.

\section{Conclusion}

Our finding of a higher prevalence of known glaucoma among patients undergoing ectropion or entropion surgery could suggest that early recognition of drug-induced eyelid malposition and discontinuation of therapy (if possible) would be important in an effort to avoid surgery. At the very least, patients with lower lid laxity should be informed of the increased risk of eyelid malposition when topical glaucoma therapy is initiated.

\section{Acknowledgments}

There was no financial support for this study. The abstract of this article was presented at the World Ophthalmology Congress, Tokyo, 2014, name PO 404 as a poster presentation with interim findings. The actual paper, however, has never been published.

\section{Disclosure}

The authors report no conflicts of interest in this work.

\section{References}

1. Shore J. Changes in lower eyelid resting position, movement and tone with age. Am J Ophthalmol. 1985;99(4):414-423.

2. Servat JJ, Bernardino CR. Effects of common topical antiglaucoma medications on the ocular surface, eyelids and periorbital tissue. Drugs Aging. 2011;28(4):267-282.

3. Aristodemou P, Baer R. Reversible cicatricial ectropion precipitated by topical brimonidine eye drops. Ophthal Plast Reconstr Surg. 2008; 24(1):57-58.

4. Bartley GB. Reversible lower eyelid ectropion associated with dipivefrin [letter]. Am J Ophthalmol. 1991;111(5):650-651.

5. Britt MT, Burnstine MA. Iopidine allergy causing lower eyelid ectropion progressing to cicatricial entropion [letter]. Br J Ophthalmol. 1999; 83(8):992-993.

6. Hegde V, Robinson R, Dean F, Mulvihill HA, Ahluwalia H. Druginduced ectropion: what is best practice? Ophthalmology. 2007;114(2): 362-366.

7. European Glaucoma Society. Chapter 2: Classification and terminology. Terminology and Guidelines for Glaucoma. Savona: Dogma; 2003:5-8.

8. Budenz DL, Barton K, Whiteside-de Vos J, et al. Prevalence of glaucoma in an urban West African population: the Tema Eye Survey. JAMA Ophthalmol. 2013;18:1-8.

9. Wright M, Bell D, Scott C, Leatherbarrow B. Everting sutures correction of lower lid involutional entropion. Br J Ophthalmol. 1999; 83(9):1060-1063.

10. Altieri M, Iester M, Harman F, et al. Comparison of three techniques for repair of involutional lower lid entropion: a three-year follow-up study. Ophthalmologica. 2003;217(4):265-272.

11. Altieri M, Kingston AE, Bertagno R, Altieri G. A modified technique of retractors plication in entropion repair: a 4-year follow-up study. Can J Ophthalmol. 2004;39:650-655.

12. Vallabhanath P, Carter SR. Ectropion and entropion. Curr Opin Ophthalmol. 2002;11(5):345-351.

13. Altieri M, Ferrari E. Do prostaglandin analogs affect eyelid position and motility? J Ocul Pharmacol Ther. 2011;27(5):511-517.

14. Damasceno RW, Osaki MH, Dantas PE, Belfort R Jr. Involutional entropion and ectropion of the lower eyelid: prevalence and associated risk factors in the elderly population. Ophthal Plast Reconstr Surg. 2011; 27(5):317-320.
Clinical Interventions in Aging

\section{Publish your work in this journal}

Clinical Interventions in Aging is an international, peer-reviewed journal focusing on evidence-based reports on the value or lack thereof of treatments intended to prevent or delay the onset of maladaptive correlates of aging in human beings. This journal is indexed on PubMed Central, MedLine,

\section{Dovepress}

CAS, Scopus and the Elsevier Bibliographic databases. The manuscript management system is completely online and includes a very quick and fair peer-review system, which is all easy to use. Visit http://www.dovepress. com/testimonials.php to read real quotes from published authors. 